\title{
Fracionamento químico da matéria orgânica e características de ácidos húmicos de solos com horizonte a antrópico da amazônia (Terra Preta)
}

\begin{abstract}
Tony Jarbas Ferreira CUNHA ${ }^{1}$; Beata Emoke MADARI ${ }^{2}$; Vinicius de Melo BENITES ${ }^{3}$; Luciano Pasqualoto CANELLAS $^{4}$; Etelvino Henrique NOVOTNY33; Rondinele de Oliveira MOUTTA ${ }^{3}$; Patrick Marques TROMPOWSKY'; Gabriel de Araújo SANTOS ${ }^{6}$

RESUMO

Os solos da Amazônia que possuem horizonte A antrópico $(\mathrm{Au})$ apresentam elevada fertilidade natural geralmente atribuída ao teor elevado de matéria orgânica e à sua elevada reatividade. Neste estudo foram quantificadas as substâncias húmicas e caracterizados os ácidos húmicos (AH) extraídos da camada 0-20 cm de solos com horizonte Au do estado do Amazonas (Terra Preta de Índio) sob floresta e cultivo agrícola. Também foram investigadas amostras de solos adjacentes sem o horizonte antrópico. A caracterização dos AH foi realizada através de análise termogravimétrica, análise da composição elementar e quantificação dos grupos funcionais (total, carboxílica e fenólica). Os horizontes A de solos antropogênicos apresentaram maior teor de carbono total, comparados aos de solos adjacentes. Entre as frações húmicas, a mais abundante foi a humina. A fração de ácidos húmicos (AH) foi a dominante das frações alcalino-solúveis nos solos estudados. Os AH dos solos antropogênicos tanto sob floresta como sob cultivo agrícola apresentaram maior grau de humificação quando comparados aos $\mathrm{AH}$ dos solos sem o horizonte Au. O cultivo agrícola também teve efeito na estabilidade e reatividade dos $\mathrm{AH}$ nos solos antropogênicos.
\end{abstract}

\section{PALAVRAS-CHAVE}

Solos antropogênicos, Amazônia, termogravimétria, composição elementar, acidez.

\section{Chemical fractionation of organic matter and humic acid characteristic in anthropogenic dark earth soils of brazilian amazonic region}

\begin{abstract}
Amazonian dark earth soils that have anthropogenic A horizon (Au) present high natural fertility usually attributed to their high organic matter content and to their higher reactivity. In this study humic substances were quantified and the humic acids $(H A)$ extracted from the $0-20 \mathrm{~cm}$ layer of Amazonian dark earth soils (Terra Preta do Indio) under forest and agricultural use were characterized. Adjacent soils with no Au horizon were also investigated. The HA were characterized through the thermogravimetric analysis, elemental composition analysis and characterization of functional groups (total, carboxylic, and phenolic). The A horizon of the anthropogenic soils presented higher total carbon level compared to adjacent soils. Among the humic fractions, the humin was the most abundant. The humic acid fraction (HA) was the dominant among the alkaline soluble fractions. The humic acids of the anthropogenic soils, both under forest and cultivation, showed higher humification degree compared to the non-anthropogenic soils. The agricultural cultivation affected the humic acids stability and reactivity in the anthropogenic soils.
\end{abstract}

\section{KEY-WORDS}

Amazonian dark earth soils, thermogravimetry, elemental composition, acidity.

\footnotetext{
${ }^{1}$ Embrapa Semi-Árido, CEP 56302-970, BR 428, Km 152, CX. Postal 23, Petrolina-PE, Brasil. e-mail: tony@cpatsa.embrapa.br ${ }^{2}$ Embrapa CNPAF, Rodovia Goiânia-Nova Veneza, Km 12, Cx. Postal 179. e-mail: madari@cnpaf.embrapa.br

${ }_{3}^{3}$ Embrapa Solos, Rua Jardim Botânico 1024, Rio de Janeiro-RJ. e-mail: vinicius@cnps.embrapa.br, etelvino@cnps.embrapa.br ${ }^{4}$ Universidade Estadual do Norte Fluminense-UENF, Av. Alberto Lamego, 2000, CEP 28013-000, Campos dos Goytacazes-RJ. e-mail:canellas@uenf.br ${ }^{5}$ Universidade Federal de Viçosa-UFV, CEP 36571-000, Viçosa-MG. patrick.marques@globo.com ${ }^{6}$ Universidade Federal Rural do Rio de Janeiro-UFRRJ, BR465, KM 47, Seropédica-RJ. e-mail: gasantos@ufrrj.br
} 


\section{INTRODUÇÃO}

A maior parte dos solos agricultáveis na Região Amazônica é de reação ácida, com baixa capacidade de troca catiônica (CTC) e baixa fertilidade. O solo é, portanto, um fator limitante para a produtividade e sustentabilidade de sistemas de produção agrícola. Nesta mesma região, ocorrem solos com horizonte A antrópico $(\mathrm{Au})$ de coloração escura, popularmente denominados "Terra Preta de Índio" (TPI) ou terra preta arqueológica (EMBRAPA, 1999; Cunha, 2005). Estes solos geralmente apresentam alta fertilidade, com elevados teores de $\mathrm{P}, \mathrm{Ca}, \mathrm{Mg}$, Zn, Mn, e com teor elevado de matéria orgânica estável (Kern \& Kämpf, 1989). Várias hipóteses foram aventadas sobre os processos de formação das TPI, mas a mais aceita atualmente diz que as TPI teriam sido formadas não intencionalmente pelo homem pré-colombiano (Woods \& McCann, 2001).

O estudo da matéria orgânica em agroecossistemas brasileiros é um tema estratégico para que se alcance a sustentabilidade da agricultura em ecossistemas tropicais e de preservação ambiental. Todavia, no que concerne aos solos possuidores de horizonte $\mathrm{A}$ antrópico, muitas questôes estão ainda por serem respondidas, principalmente quanto a sua origem, distribuição e características da sua matéria orgânica.

A elevada fertilidade e principalmente a sustentabilidade da fertilidade das TPI, pelo nosso conhecimento atual, é atribuída ao elevado nível de matéria orgânica e as suas propriedades físicoquímicas como, por exemplo, a elevada reatividade das fraçōes húmicas. Uma proporção significativa, até $35-45 \%$, do carbono orgânico nas TPI está na forma de carbono pirogênico comparado a 14\% em solos circunvizinhos sem horizonte Au, e 48 a 59\% deste encontra-se na fração leve da matéria orgânica do solo (Glaser et al., 2000). A natureza do carbono pirogênico (particulada, livre, protegida e em complexos organo-minerais) indica o papel significativo na formação das propriedades físicas (estruturação, retenção de água etc.) destes solos, que afeta, positivamente, sua fertilidade.

A contribuição do carbono pirogênico à fertilidade e à sustentabilidade da fertilidade do solo é atribuída principalmente à sua composição e estrutura molecular que foi investigado por vários autores (Golchin et al., 1997; Schmid et al., 2002). Foi observado que a proporção de carbono na matéria orgânica das TPI foi elevada em estruturas aromáticas, comparado com latossolos. Ao mesmo tempo, a proporção dos grupos carboxílicos nas TPI não foi diminuída comparada com latossolos, indicando que o carbono das Terras Pretas tem características de substâncias orgânicas mais estáveis e ao mesmo tempo reativas (Zech et al., 1990).

A fertilidade dos solos geralmente é fortemente relacionada às características moleculares da fração alcalino solúvel do carbono orgânico, as substâncias húmicas (SH). Esta fração, ao mesmo tempo é dinâmica, refletindo mudanças no uso do solo, e também é uma das frações responsáveis pela acumulação da matéria orgânica no solo. Em relaçãa à TPI existem poucos estudos sobre a caracterização molecular das substâncias húmicas (Zech et al., 1990; Lima, 2001). Uma questão aberta é se o elevado teor de carbono pirogênico afeta as propriedades das $\mathrm{SH}$ de maneira que através disso possa afetar a fertilidade das TPI. O efeito do uso agrícola das TPI nas propriedades da fração humificada da matéria orgânica é também desconhecido.

O objetivo deste trabalho foi quantificar as substâncias húmicas e estudar as características de estabilidade e reatividade dos ácidos húmicos de solos com horizonte $\mathrm{A}$ antrópico $(\mathrm{Au}) \mathrm{de}$ diversas regiōes do Estado do Amazonas e observar o efeito de uso agrícola sobre estas propriedades. Foram determinadas a sua composição elementar, comportamento termogravimétrico e grupamentos funcionais.

\section{MATERIAL E MÉTODOS}

Foram selecionadas áreas de ocorrência de solos antropogênicos (latossolo amarelo coeso antrópico) nas regiōes de Humaitá, Lábrea, Apuí, Novo Aripuanã, Manicoré e Manaus, no estado do Amazonas (Fig. 1). Nestes solos foram realizadas amostragens na profundidade de $0-20 \mathrm{~cm}$ coletando-se doze subamostras para composição da amostra completa (Lemos \& Santos, 1996). As mesmas foram misturadas, secas ao ar e passadas em peneira de dois milímetros. As áreas de estudo foram selecionadas em função dos diferentes usos, abrangendo áreas sob floresta e áreas cultivadas. Também foram selecionados solos sob cobertura de floresta e adjacentes a algumas manchas de solos antropogênicos, para fins de comparação. As amostras foram agrupadas como SAF: Solos antropogênicos sob floresta $(\mathrm{n}=$ 06), SAC: solos antropogênicos sob cultivo agrícola $(n=12)$, e SNAF: solos não antropogênicos sob floresta $(\mathrm{n}=04)$ (Tabela $01)$. As diferenças entre médias dos grupos foram analisadas pelo teste de Tukey a $5 \%$.

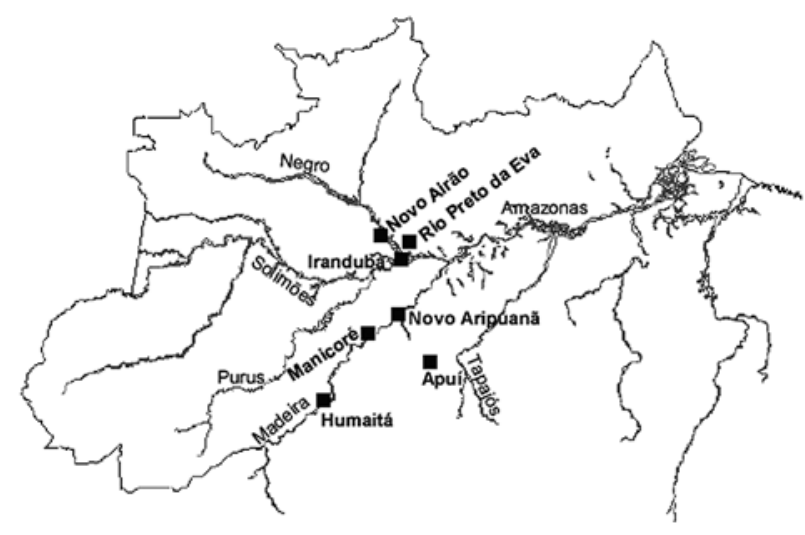

Figura 1 - Localização dos municípios estudados. 


\section{QUANTIFICAÇÃO DO CARBONO TOTAL E DAS SUBSTÂNCIAS HÚMICAS}

O teor de carbono total do solo foi determinado via combustão a $925^{\circ} \mathrm{C}$ usando analisador elementar Perkin Elmer CHN/O Analyser 2400 Series II. O fracionamento químico quantitativo das substâncias húmicas $(\mathrm{SH})$ foi realizado segundo Benites et al. (2003), com três repetiçôes, sendo obtidas as fraçôes huminas $(\mathrm{H})$, ácidos húmicos $(\mathrm{AH})$ e ácidos fúlvicos $(\mathrm{AF})$, conforme segue: amostras de solo foram tratadas com $\mathrm{NaOH}$ 0,1 mol. $^{-1} \mathrm{em}$ atmosfera de $\mathrm{N}_{2}$, agitadas manualmente e deixadas em repouso por $24 \mathrm{~h}$. Após esta etapa, as mesmas forma centrifugadas a $5.000 \mathrm{~g}$ por 30 minutos. Este procedimento foi repetido por mais duas vezes. $\mathrm{O} \mathrm{pH}$ do extrato alcalino foi ajustado para 1,0 pela adição de $\mathrm{H}_{2} \mathrm{SO}_{4}$ e deixado para decantar durante $18 \mathrm{~h}$. O material acidificado foi filtrado e teve o volume aferido para $50 \mathrm{ml}$ com água destilada (fração de ácidos fúlvicos). Sobre o precipitado foram adicionados $\mathrm{NaOH} 0,1$ mol. $^{-1}$ até a lavagem completa do filtro e foram aferidos o volume para $50 \mathrm{ml}$ usando água destilada (fração ácidos húmicos). O material restante nos tubos de centrífuga foi considerado como a fração humina. A determinação quantitativa de carbono nos extratos das fraçôes ácidos fúlvicos, ácidos húmicos e humina foram feitas através da oxidaçāo do C com dicromato de potássio e titulação do excesso,

Tabela 1 - Local de amostragem, solo correspondente e agrupamento para análise estatística.

\begin{tabular}{|c|c|c|c|c|}
\hline Amostra & Solo & Uso & Grupo & Localização \\
\hline 01 & Antropogênico & Floresta & SAF & Humaitá \\
\hline 02 & Antropogênico & Floresta & SAF & Novo Aripuanã \\
\hline 03 & Antropogênico & Floresta & SAF & Apuí \\
\hline 04 & Antropogênico & Floresta & SAF & Apuí \\
\hline 06 & Antropogênico & Floresta & SAF & Iranduba \\
\hline 07 & Antropogênico & Floresta & SAF & Rio Preto da Eva \\
\hline 10 & Antropogênico & Cultivado & SAC & Humaitá \\
\hline 11 & Antropogênico & Cultivado & SAC & Novo Aripuanã \\
\hline 13 & Antropogênico & Cultivado & SAC & Apuí \\
\hline 14 & Antropogênico & Cultivado & SAC & Rio Preto da Eva \\
\hline 15 & Antropogênico & Cultivado & SAC & Rio Preto da Eva \\
\hline 16 & Antropogênico & Cultivado & SAC & Novo Airão \\
\hline 17 & Antropogênico & Cultivado & SAC & Rio Preto da Eva \\
\hline 18 & Antropogênico & Cultivado & SAC & Rio Preto da Eva \\
\hline 19 & Antropogênico & Cultivado & SAC & Iranduba \\
\hline 20 & Antropogênico & Cultivado & SAC & Iranduba \\
\hline 21 & Antropogênico & Cultivado & SAC & Novo Airão \\
\hline 22 & Antropogênico & Cultivado & SAC & Açutuba \\
\hline 26 & Não Antropogênico & Floresta & SNAF & Manicoré \\
\hline 27 & Não Antropogênico & Floresta & SNAF & Humaitá \\
\hline 28 & Não Antropogênico & Floresta & SNAF & Manicoré \\
\hline 29 & Não Antropogênico & Floresta & SNAF & Humaitá \\
\hline
\end{tabular}

com sulfato ferroso amoniacal de acordo com Yeomans \& Bremner (1988). Dos teores de cada fração de SH foi calculada a relação $\mathrm{AH} / \mathrm{AF}$ e a relação entre as fraçôes no extrato alcalino $(\mathrm{AF}+\mathrm{AH}=\mathrm{EA})$ e humina $(\mathrm{H})$, obtendo-se a relação $\mathrm{EA} / \mathrm{H}$ (Benites et al., 2003).

\section{EXTRAÇÃO, FRACIONAMENTO E PURIFICAÇÃO DOS AH PARA FINS ANALÍTICOS}

A extração e o fracionamento das $\mathrm{SH}$ para obtenção dos $\mathrm{AH}$, bem como sua purificação, foram realizados conforme método da Sociedade Internacional de Substâncias Húmicas (IHSS), cujo protocolo metodológico encontra-se descrito em Swift (1996). Houve uma modificação em relação ao protocolo da IHSS, onde houve o pré-tratamento da amostra de solo com uma solução de $\mathrm{HCl} 1$ mol..$^{-1}$, o $\mathrm{pH}$ foi ajustado entre 1 e 2 para remoção de metais adsorvidos e a utilização do $\mathrm{K}^{+}$para remoção dos sólidos em suspensão (Machado, 1999). As amostras purificadas foram lavadas com $200 \mathrm{ml} \mathrm{de} \mathrm{HCl} 0,01$ mol. $\mathrm{l}^{-1}$, centrifugadas a 5.000 $\mathrm{g}$ e transferidas para sacolas de celofane de aproximadamente $100 \mathrm{ml}$, dializadas e liofilizadas.

\section{ANÁLISE TERMOGRAVIMÉTRICA E DETERMINAÇÃO DO CONTEÚDO DE CINZAS DOS ÁCIDOS HÚMICOS}

As curvas de termodecomposição das amostras de $\mathrm{AH}$ foram obtidas em um analisador termogravimétrico TGA-50 SHIMADZU utilizando-se 3,3 $\pm 0,1 \mathrm{mg}$ de amostras em ambiente estático ao ar conforme Benites et al. (2005). O peso inicial foi estabilizado a $30^{\circ} \mathrm{C}$ e a curva de aquecimento foi de 5 ${ }^{\circ} \mathrm{C} \cdot \mathrm{min}^{-1}$ até $105^{\circ} \mathrm{C}$ com um tempo de espera de $10 \mathrm{~min}$, seguido de aquecimento a $5^{\circ} \mathrm{C} \min ^{-1}$ até $650^{\circ} \mathrm{C}$, ou até 600 ${ }^{\circ} \mathrm{C}$, quando detectado o final da queima pela estabilização do peso do resíduo. O processo consumiu em média 2 horas e 30 minutos por amostra, além de 30 minutos adicionais para o resfriamento do aparelho. A perda de peso até $105^{\circ} \mathrm{C}$, incluído o tempo de espera de dez minutos, foi considerada como umidade da amostra. O resíduo ao final da queima foi considerado como igual ao teor de cinzas. $O$ índice termogravimétrico (ITG) foi calculado pela razão entre a perda de peso por ignição (PPI) no segundo evento $\left(350-650^{\circ} \mathrm{C}\right)$ e a perda de peso por ignição (PPI) no primeiro evento $\left(105-350^{\circ} \mathrm{C}\right)$, conforme procedimento descrito em Benites et al. (2005).

\section{ANÁLISE DA COMPOSIÇÃO ELEMENTAR DOS ÁCIDOS HÚMICOS}

A composição elementar dos $\mathrm{AH}$ foi determinada via combustão a $925^{\circ} \mathrm{C}$ em analisador elementar Perkin Elmer CHN/O Analyser 2400 Series II. Os valores de carbono (C), hidrogênio $(\mathrm{H})$ e nitrogênio $(\mathrm{N})$ foram corrigidos para base seca e livre de cinzas, utilizando-se as informaçóes obtidas pelos dados da análise termogravimétrica. $\mathrm{O}$ teor de oxigênio $(\mathrm{O})$ foi determinado por diferença a partir dos dados corrigidos. Também foram calculadas as razões atômicas: $\mathrm{H} / \mathrm{C}, \mathrm{C} / \mathrm{N}, \mathrm{O} / \mathrm{C} \mathrm{e} \mathrm{O} / \mathrm{H}$. 


\section{ACIDEZ TOTAL, ACIDEZ CARBOXÍLICA (COOH) E ACIDEZ FENÓLICA (OH) DOS ÁCIDOS HÚMICOS}

As diferentes formas de acidez foram determinadas conforme Schnitzer \& Gupta (1965). A acidez total foi medida colocandose de 50 a $100 \mathrm{mg}$ de amostras de $\mathrm{AH}$ em frascos Kitasato, onde foram adicionados $20 \mathrm{ml} \mathrm{de} \mathrm{Ba}(\mathrm{OH})_{2}$ a 0,125 mol. $^{-1}$, preparado com água livre de $\mathrm{CO}_{2}$ dissolvido. Simultaneamente, foi realizada uma prova em branco somente com $20 \mathrm{ml} \mathrm{de} \mathrm{Ba}(\mathrm{OH})_{2} 0,125$ mol. $1^{-1}$. O ar dos frascos foi deslocado pela passagem de $\mathrm{N}_{2}$ e o sistema submetido à agitação por $24 \mathrm{~h}$ em temperatura ambiente. A suspensão foi filtrada e o resíduo lavado com água fervida livre de $\mathrm{CO}_{2}$. O filtrado foi titulado potenciometricamente até $\mathrm{pH}$ $8,4 \mathrm{com} \mathrm{HCl} \mathrm{0,5}$ mol. $^{-1}$. A acidez carboxílica foi determinada colocando amostras de 50 a $100 \mathrm{mg}$ de $\mathrm{AH}$ em frascos de Erlemeyer de $125 \mathrm{ml}$, adcionando $40 \mathrm{ml}$ de água sem $\mathrm{CO}_{2}$ e 10 $\mathrm{ml} \mathrm{de} \mathrm{Ca}(\mathrm{OAc})_{2} 1 \mathrm{~mol}^{-\mathrm{I}^{-1}}$. Simultaneamente, foi conduzida uma prova em branco somente com $40 \mathrm{ml}$ de água livre de $\mathrm{CO}_{2}$ e 10 $\mathrm{ml}$ de $\mathrm{Ca}(\mathrm{OAc})_{2} 1 \mathrm{~mol}^{-1} \mathrm{I}^{-1}$. O sistema foi submetido à agitação por $24 \mathrm{~h} \mathrm{em} \mathrm{temperatura} \mathrm{ambiente.} \mathrm{A} \mathrm{suspensão} \mathrm{foi} \mathrm{filtrada} \mathrm{e} \mathrm{o}$ resíduo lavado com água livre de $\mathrm{CO}_{2}$. O filtrado foi titulado potenciometricamente até $\mathrm{pH} 9,8 \mathrm{com} \mathrm{NaOH} 0,1$ mol. $^{-1} \mathrm{~A}$ acidez fenólica foi obtida por diferença entre a acidez total e a acidez carboxílica.

\section{RESULTADOS E DISCUSSÃO}

\section{QUANTIFICAÇ̃̃O DO CARBONO TOTAL E FRACIONAMENTO DA MATÉRIA ORGÂNICA HUMIFICADA.}

Os resultados obtidos com o fracionamento da matéria orgânica humificada das amostras de solo estão apresentados na Tabela 2.

Foi possível observar diferenças significativas no teor de carbono total das amostras de solo dos grupos $\mathrm{SAF}$ e SAC quando comparados ao do grupo SNAF. Os valores observados neste estudo foram coerentes com observações realizadas em outros solos antropogênicos (Sombroek, 1966; Smith, 1980; Kern \& Kämpf, 1989).

O conteúdo elevado de carbono nos solos antropogênicos, mesmo estando em ambiente favorável à decomposição e

Tabela 2 - Valores médios dos teores das frações das substâncias húmicas (SH), carbono total, teores relativos das $\mathrm{SH}$ ao carbono total, e as relações entre frações, agrupados por tipo uso e de solo.

\begin{tabular}{|c|c|c|c|c|c|c|c|c|c|}
\hline Grupo & $\begin{array}{c}\text { Carbono } \\
\text { total }\end{array}$ & AF & $\mathrm{AH}$ & $\mathrm{H}$ & $\mathrm{AF}$ & $\mathrm{AH}$ & H & $\frac{\mathrm{AH}}{\mathrm{AF}}$ & $\frac{E A}{H}$ \\
\hline & \multicolumn{4}{|c|}{-- g kg$^{-1}$-..- } & \multicolumn{3}{|c|}{----- \% -------- } & & \\
\hline SAF & $44,6 a$ & $6,3 a$ & $13,9 a$ & $20,1 a$ & $15 \mathrm{a}$ & $31 a$ & $45 \mathrm{a}$ & $2,1 \mathrm{a}$ & $1,06 a$ \\
\hline SAC & $44,7 a$ & $5,1 \mathrm{a}$ & $14,6 \mathrm{a}$ & $19,3 a$ & $11 \mathrm{a}$ & $33 a$ & $43 a$ & $3,0 \mathrm{a}$ & $1,08 a$ \\
\hline SNAF & $37,9 \mathrm{~b}$ & $5,9 a$ & $7,1 \mathrm{~b}$ & $18,6 a$ & $16 a$ & $19 b$ & $49 a$ & $1,2 b$ & $0,72 a$ \\
\hline
\end{tabular}

Médias seguidas de letras diferentes na mesma coluna diferem significativamente a $5 \%$ pelo teste de Tukey. AF: ácidos fúlvicos; AH: ácidos húmicos; $\mathrm{H}$ : huminas; $\mathrm{EA}$ : extrato alcalino = AF + AH; EA/H: (AF + AH)/H. SAC: solos antropogênicos sob floresta; $S A C$ solos antropogênicos sob cultivo; SNAF: solos não antropogênicos sob floresta. lixiviação, pode ser atribuído à formação de complexos matéria orgânica-cálcio de alta estabilidade (teor médio de cálcio trocável nas TP foi de $7,4 \mathrm{cmol} \cdot \mathrm{kg}^{-1}$ (Cunha, 2005), ou ainda à própria composição da matéria orgânica rica em carbono pirogênico (carvão) adicionada ao solo pela atividade dos povos indígenas pré-colombianos (Glaser, 2000).

Nos solos com horizonte Au (SAF e SAC), independente do uso, foram observados valores relativos mais elevados para os $\mathrm{AH}$ em comparação com solos sem a presença deste horizonte. Além disso, a concentração dos $\mathrm{AH}$, comparado com os $\mathrm{AF}$, foi significativamente maior nos solos antropogênicos $(\mathrm{AH} / \mathrm{AF}=$ 2,1 e 3,0 para os grupos SAF e SAC, respectivamente) do que nos solos não antropogênicos $(\mathrm{AH} / \mathrm{AF}=1,2)$. A fração $\mathrm{H}$ foi a humificada dominante em todas as amostras. Os teores em g. $\mathrm{kg}^{-}$ ${ }^{1}$ das frações húmicas obedeceram o mesmo comportamento da sua proporção relativa ao carbono total.

Estudando a composição das $\mathrm{SH}$ de solos antropogênicos da Amazônia, Lima (2001) também verificou que nestes solos houve uma predominância das frações mais estáveis $(\mathrm{AH}$ e $\mathrm{H})$, com menor contribuição das fraçōes mais solúveis e móveis (AF). O autor encontrou valores para a razão $\mathrm{AH} / \mathrm{AF}$ da ordem de 14 no horizonte A de um Cambissolo Antrópico e da ordem de quatro em um Argissolo Antrópico. O predomínio da fração AH sobre a fração $\mathrm{AF}$ em solos antropogênicos, ao contrário do que se observa para os demais solos de terra firme e várzea da Amazônia, pode ser o resultado de intensa humificação e rápida mineralização de grandes quantidades de material orgânico rico em N, P e Ca incorporado ao solo (Zech et al., 1990). Por outro lado, as condiçōes bioclimáticas atuais como temperatura e precipitação elevadas, drenagem adequada e caráter eutrófico dos solos antropogênicos devem favorecer também o acúmulo de matéria orgânica mais estável à decomposição e, conseqüentemente, à manutenção de altas razões AH/AF. Foi observada menor razão $\mathrm{AH} / \mathrm{AF}$ nos solos pertencentes ao grupo $\mathrm{SNAF}$, que apresentam baixo conteúdo de bases trocáveis (Cunha, 2005), quando comparado aos grupos SAF e SAC (Tabela 2). A presença da fração AH tem sido relacionada a solos férteis, com alto conteúdo de bases trocáveis (Canellas et al., 2003).

\section{ANÁLISE TERMOGRAVIMÉTRICA}

As curvas termogravimétricas dos $\mathrm{AH}$ isolados de solos com horizonte $\mathrm{Au}$ (SAF e SAC) e de solos não antropogênicos (SNAF) estão apresentadas na Fig. 2. Os termogramas foram caracterizados por dois picos de oxidação exotérmica: o primeiro (105 e 350 $\left.{ }^{\circ} \mathrm{C}\right)$ é atribuído à degradação térmica de estruturas menos estáveis, por exemplo, polissacarídeos, descarboxilação de grupos ácidos e desidratação de estruturas alifáticas hidroxiladas (Francioso $e t$ al., 2003), e o segundo pico $\left(350\right.$ e $\left.650^{\circ} \mathrm{C}\right)$, à degradação de estruturas aromáticas e rompimento de ligaçôes C-C (Provenzano $\&$ Senesi, 1999). Aspecto semelhante (bimodal) das curvas das primeiras derivadas de termogramas de AH foi também observado por (Benites et al., 2005). 


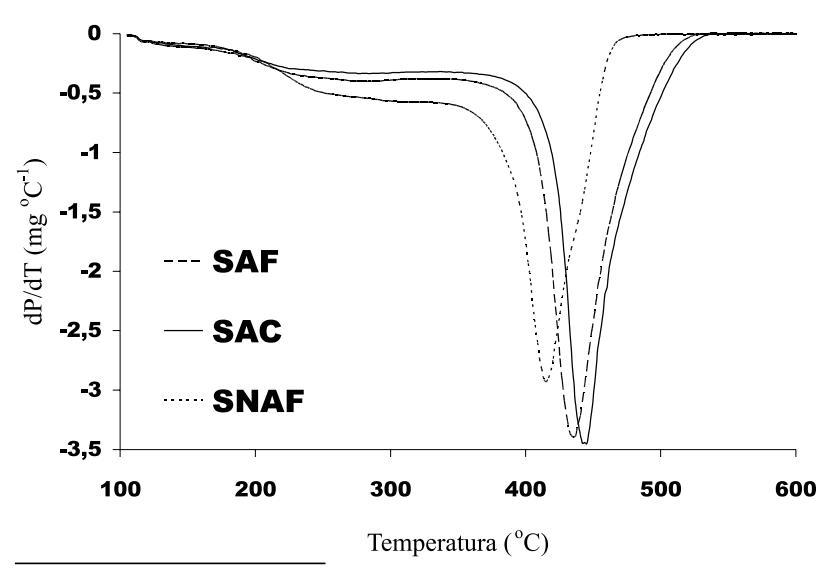

Figura 2 - Curvas médias das primeiras derivadas dos termogramas dos ácidos húmicos representativos dos solos antropogênicos sob floresta (SAF), sob cultivo (SAC) e de solos não antropogênicos (SNAF).

Os AH apresentaram variaçōes entre os diferentes grupos estudados, no que diz respeito ao percentual de perda de massa em cada evento. Na Tabela 3 estáo apresentados os valores médios referentes aos dados da análise termogravimétrica nos diferentes grupos de $\mathrm{AH}$. Os conteúdos médios de cinzas variaram de 2,4 a $8,0 \%$.

Entre os grupos SAF, SAC e SNAF foram observados que a perda de massa no primeiro evento foi maior para o grupo SNAF, sugerindo que os $\mathrm{AH}$ deste grupo são menos resistentes à degradação térmica, possivelmente, por apresentarem menor conteúdo de estruturas aromáticas policíclicas (Benites et al., 2005) típicas de substâncias húmicas derivadas de carbono pirogênico (Kramer et al., 2004), o que se confirma pelo menor índice termogravimétrico (ITG), observado para os AH deste grupo.

Tabela 3 - Dados médios da análise termogravimétrica dos ácidos húmicos.

\begin{tabular}{|c|c|c|c|c|}
\hline \multirow[t]{2}{*}{ Grupo } & \multirow{2}{*}{$\begin{array}{c}\text { Umidade } \\
\%\end{array}$} & \multicolumn{2}{|c|}{ PPI } & \multirow[t]{2}{*}{ ITG } \\
\hline & & $105-350^{\circ} \mathrm{C}$ & $350-650^{\circ} \mathrm{C}$ & \\
\hline SAF & $4,75 \mathrm{~b}$ & $22,37 \mathrm{~b}$ & $64,90 \mathrm{a}$ & $3,0 \mathrm{~b}$ \\
\hline SAC & $7,01 \mathrm{a}$ & $21,09 \mathrm{~b}$ & 69,49 a & $3,3 \mathrm{a}$ \\
\hline SNAF & $6,78 \mathrm{a}$ & $26,47 \mathrm{a}$ & $61,47 \mathrm{a}$ & $2,3 \mathrm{c}$ \\
\hline
\end{tabular}

Médias seguidas de letras diferentes na mesma coluna diferem significativamente a $5 \%$ pelo teste de Tukey; PPI: perda de peso por ignição; ITG: índice termogravimétrico. solos antropogênicos sob floresta; SAC: solos antropogênicos sob cultivo; SNAF: solos não antropogênicos sob floresta.
$\mathrm{O}$ fato dos $\mathrm{AH}$ originados dos solos com horizonte $\mathrm{Au}$ apresentarem perda relativa de massa maior no segundo evento (maior ITG) em relação ao grupo SNAF, pode estar relacionado à maior proporção de grupamentos aromáticos altamente condensados presentes nestes AH (Kramer et al., 2004). A alta resistência de $\mathrm{AH}$ à oxidação térmica, também foi observado por Benites et al. (2005), estudando AH extraídos de solos de campos de altitude. $\mathrm{O}$ autor atribuiu este comportamento à origem pirogênica e à ocorrência de núcleos aromáticos policíclicos nos mesmos.

\section{COMPOSIÇÃO ELEMENTAR}

Os resultados da análise de composição elementar dos $\mathrm{AH}$ e as razóes atômicas $(\mathrm{H} / \mathrm{C}, \mathrm{O} / \mathrm{Ce} \mathrm{C} / \mathrm{N})$ calculadas estão apresentados na Tabela 4. As razões atômicas têm sido utilizadas indiretamente para descrever o grau de condensação, a fonte de matéria orgânica, as transformaçōes diagenéticas, bem como as condiçōes ambientais sob as quais elas foram formadas (Rice \& MacCarthy, 1991), além de serem um indicador simples do comportamento químico das substâncias húmicas (Canellas et al., 1999). A razão H/C é considerada como índice de aromaticidade (Stevenson, 1994). A razão atômica $\mathrm{O} / \mathrm{C}$ permite estimar a abundância de oxigênio contido em grupos funcionais (Bravard \& Righi, 1991). A relação $\mathrm{C} / \mathrm{N}$ indica o grau de incorporação do $\mathrm{N}$ na estrutura das $\mathrm{SH}$ e o grau de humificação (Stevenson, 1994).

Os AH do grupo SNAF apresentaram maior razão H/C e menor relação $\mathrm{C} / \mathrm{N}$ que os grupos $\mathrm{SAF}$ e $\mathrm{SAC}$. Estes resultados sugerem, em concordância com os resultados da análise termogravimétrica, que os $\mathrm{AH}$ dos solos com horizonte $\mathrm{Au}$ contém, relativamente às outras partes (lábeis e alifáticas) na sua estrutura molecular, mais estruturas aromáticas. As estruturas mais lábeis, que foram mais abundantes no grupo SNAF, também foram as que contiveram o maior teor de $\mathrm{N}$ comparado com $\mathrm{SAF}$ e $S A C$, como sugerido pela razão $\mathrm{C} / \mathrm{N}$. Por outro lado, o grupo $\mathrm{SAF}$ contém mais $\mathrm{O}$ relativo ao $\mathrm{C}$, o que sugere uma maior abundância de grupos funcionais ácidos, em relação ao grupo SNAF. Os resultados da análise elementar (razão H/C), junto com os da análise termogravimétrica (ITG) indicaram que os $\mathrm{AH}$ dos solos antropogênicos foram mais estáveis e apresentaram maior grau de humificação, em comparação com os $\mathrm{AH}$ dos solos sem horizonte Au. A razão $\mathrm{H} / \mathrm{C}$ é considerada como índice de aromaticidade (Stevenson, 1994). Benites et al. (2005) utilizou o índice ITG para avaliar o grau de humificação de substâncias

Tabela 4 - Composição elementar, razões atômicas e cinzas entre os grupos de ácidos húmicos.

\begin{tabular}{|c|c|c|c|c|c|c|c|c|c|}
\hline \multirow[t]{2}{*}{ Grupo } & \multirow[t]{2}{*}{$C$} & \multirow[t]{2}{*}{$\mathrm{H}$} & \multirow{2}{*}{$\begin{array}{l}\mathrm{N} \\
---\end{array}$} & \multirow[t]{2}{*}{0} & \multicolumn{4}{|c|}{ Razão atômica } & \multirow{2}{*}{$\begin{array}{c}\text { Cinzas } \\
\%\end{array}$} \\
\hline & & & & & $\mathrm{H} / \mathrm{C}$ & $0 / C$ & $0 / H$ & $\mathrm{C} / \mathrm{N}$ & \\
\hline SAF & $598,0 \mathrm{~b}$ & $44,0 \mathrm{~b}$ & $60,0 \mathrm{~b}$ & $298,0 a$ & $0,90 \mathrm{~b}$ & $0,40 \mathrm{a}$ & $6,87 a$ & $11,70 a$ & $8,00 a$ \\
\hline SAC & $627,0 \mathrm{a}$ & $37,0 \mathrm{c}$ & $59,0 \mathrm{~b}$ & $277,0 \mathrm{~b}$ & $0,70 \mathrm{c}$ & $0,30 \mathrm{~b}$ & $7,70 \mathrm{a}$ & $12,60 \mathrm{a}$ & $2,40 \mathrm{~b}$ \\
\hline SNAF & $596,0 \mathrm{~b}$ & $56,0 \mathrm{a}$ & $80,0 a$ & $268,0 \mathrm{~b}$ & $1,10 \mathrm{a}$ & $0,30 \mathrm{~b}$ & $4,76 b$ & $8,80 \mathrm{~b}$ & $5,30 \mathrm{a}$ \\
\hline
\end{tabular}

Médias seguidas de letras diferentes na mesma coluna diferem significativamente a 5\% pelo teste de Tukey. solos antropogênicos sob floresta; SAC: solos antropogênicos sob cultivo; SNAF: solos não antropogênicos sob floresta. 
húmicas. Foi possível observar uma estreita correlação entre a razão H/C, considerada como índice de aromaticidade (Stevenson, 1994), e o ITG sugerido como índice de estabilidade por Benites et al. (2005) (Fig. 3). Quanto menor a razão atômica H/C, maior a resistência da amostra à termodegradação, indicando alta resistência de estruturas aromáticas a processos de oxidação.

Entre os $\mathrm{AH}$ dos solos com horizonte Au foi possível verificar que o cultivo diminuiu o teor de $\mathrm{He} \mathrm{O}$ relativo ao $\mathrm{C}$ (razóes $\mathrm{H}$ / $\mathrm{C} \mathrm{e} \mathrm{O/C} \mathrm{foram} \mathrm{menores} \mathrm{para} \mathrm{o} \mathrm{SAC).} \mathrm{De} \mathrm{acordo} \mathrm{com} \mathrm{Stevenson}$ (1994), quanto maior o estágio de humificação das SH, maior é o teor de $\mathrm{C}$ e menor o de $\mathrm{H}$ (razão H/C menor). Dessa forma, pode-se inferir que o cultivo dos solos antropogênicos favoreceu condições no solo que resultaram no aumento do grau de humificação dos $\mathrm{AH}$. O aumento do grau de humificação com o cultivo também foi reportado por Bayer et al. (2002) e Pérez et al. (2004). O maior teor de $\mathrm{C}$ e o menor conteúdo de $\mathrm{O}$ nos $\mathrm{AH}$ do grupo SAC, em comparação ao SAF (razão O/C menor para $S A C)$, corrobora e pode explicar a maior resistência à termodegradação dos $\mathrm{AH}$ extraídos dos solos antropogênicos sob cultivo (SAC) (Tabela 3).

A relação $\mathrm{C} / \mathrm{N}$ indica o grau de incorporação do $\mathrm{N}$ na estrutura de $\mathrm{SH}$ e o grau de humificação. Uma alta relação $\mathrm{C} / \mathrm{N}$ indica baixa incorporação de $\mathrm{N}$ e baixo grau de humificação. A relação C/N nos grupos SAF e SAC encontram-se dentro da faixa que indica estabilidade biológica $(10-12)$, (Steverson,(1994). Isso sugere pequena mineralização secundária da matéria orgânica destes solos (Rosell et al., 1989). Por outro lado, nos AH do grupo SNAF, o valor desta relação encontra-se abaixo da faixa sugerida para estabilidade biológica, indicando que neste grupo o processo de mineralização secundária é maior, evidenciando a baixa resistência destes $\mathrm{AH}$ a este processo.

\section{ACIDEZ TOTAL, CARBOXÍLICA E FENÓLICA}

Dentre os grupamentos funcionais estudados observou-se uma maior quantidade de grupamentos carboxílicos, em relação

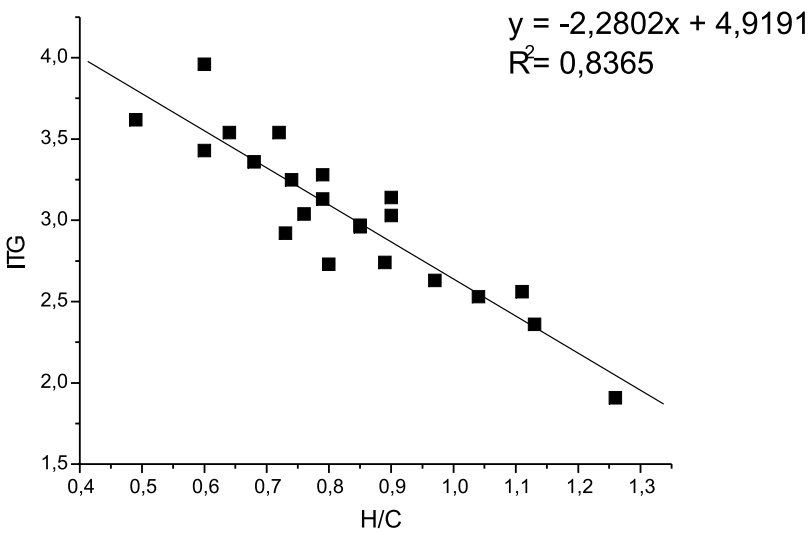

Figura 3. Correlação entre o índice termogravimétrico (ITG) e a razão atômica $\mathrm{H} / \mathrm{C}$ dos ácidos húmicos. a grupamentos fenólicos, em todos os grupos de $\mathrm{AH}$ com valores da razão $\mathrm{COOH} / \mathrm{OH}$ variando entre 2,76 e 1,44. Comportamento semelhante tem sido reportado na literatura (Schnitzer \& Khan, 1972). Isso, provavelmente, deve-se ao fato de que compostos fenólicos podem ser facilmente oxidados em ambientes aeróbios (Baldock et al., 1997).

Não foi observada diferença significativa na acidez total dos $\mathrm{AH}$ dos grupos SAF e SNAF. No entanto, AH isolados do grupo $\mathrm{SAC}$ apresentaram maior quantidade de moles de $\mathrm{H}$ disponíveis para as reações de troca iônica em relação aos AH isolados dos solos dos outros grupos, ambos sob vegetação natural (floresta).

O conteúdo de grupos carboxílicos tem sido considerado como um índice válido de humificação (Rosell et al., 1989). Observou-se que os AH originados dos solos antropogênicos (grupos SAF e SAC) apresentaram maior quantidade de grupos carboxílicos, em comparação ao grupo SNAF (Tabela 5).

Isto sugere maior grau de humificação dos $\mathrm{AH}$ dos grupos SAF e SAC, que é corroborado pelos resultados obtidos nas outras análises descritas neste trabalho. Também se observou que o aumento no grau de humificação (indicado pela diminuição da razão $\mathrm{H} / \mathrm{C}$ ) foi acompanhado pelo aumento da acidez total e carboxílica (Fig. 4).

Tabela 5 - Grupos funcionais $\left(\mathrm{cmol}_{\mathrm{c}} \cdot \mathrm{kg}^{-1}\right)$ contendo oxigênio nos ácidos húmicos.

\begin{tabular}{cccc}
\hline Grupos & Acidez Total & $\begin{array}{c}\text { Acidez Carboxilica } \\
(\mathrm{COOH})\end{array}$ & Acidez Fenólica (OH) \\
\hline SAF & $612 \mathrm{~b}$ & $435 \mathrm{a}$ & $177 \mathrm{a}$ \\
SAC & $712 \mathrm{a}$ & $454 \mathrm{a}$ & $258 \mathrm{a}$ \\
SNAF & $575 \mathrm{~b}$ & $320 \mathrm{~b}$ & $256 \mathrm{a}$ \\
\hline
\end{tabular}

Médias seguidas de letras diferentes na mesma coluna diferem significativamente a $5 \%$ pelo teste de Tukey. solos antropogênicos sob floresta; SAC: solos antropogênicos sob cultivo; SNAF: solos não antropogênicos sob floresta.

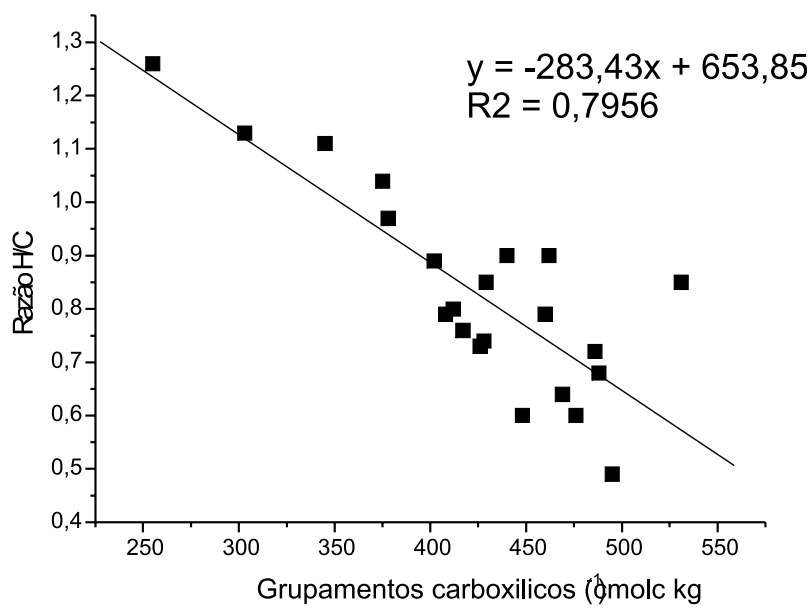

Figura 4 - Correlação entre a razão atômica $\mathrm{H} / \mathrm{C}$ e a quantidade de grupos carboxílicos dos ácidos húmicos. 
Em resumo, os AH pertencentes aos grupos SAF e SAC são potencialmente mais reativos, o que favorece as reações de troca iônica, reaçôes com colóides minerais do solo, reaçôes de complexão com cátions metálicos e maior contribuição às reações dos ciclos biogeoquímicos.

\section{CONCLUSÕES}

Os horizontes A de solos antropogênicos da Amazônia apresentaram maior teor de carbono total, comparados aos de solos adjacentes não antropogênicos que não apresentaram horizonte A antrópico. Entre as fraçôes húmicas, a mais abundante é a humina. A fração de ácidos húmicos $(\mathrm{AH})$ foi a dominante nas frações alcalino-solúveis nos solos estudados.

As principais diferenças entre os $\mathrm{AH}$ dos solos antropogênicos (SAF e SAC), em relação aos solos não antropogênicos (SNAF) foram em grau de humificação dos $\mathrm{AH}$, estando os $\mathrm{AH}$, pertencentes aos grupos $\mathrm{SAF}$ e $\mathrm{SAC}$, num estágio mais avançado de humificação. O maior grau de humificação dos $\mathrm{AH}$ de solos antropogênicos foi devido à maior concentração de estruturas estáveis (aromáticas) e reativas (grupos carboxílicos).

Diferenças foram detectadas também nas características de estabilidade e reatividade dos $\mathrm{AH}$ dos solos antropogênicos sob floresta (SAF) e cultivo (SAC). Pela análise termogravimétrica e razão $\mathrm{H} / \mathrm{C}$, os $\mathrm{AH}$ do grupo SAC mostraram mais estabilidade estrutural que pode ser devido à preservação seletiva de núcleos aromáticos. Os AH do grupo SAC também mostraram maior reatividade (acidez total) do que os pertencentes ao grupo SAF, possivelmente, devido ao ambiente mais favorável à formação ou preservação de grupos funcionais ácidos nos solos SAC.

\section{AGRADECIMENTOS}

Agradecemos à EMBRAPA Solos, à EMBRAPA Instrumentação Agropecuária, à Universidade Estadual do Norte Fluminense e à Universidade Federal Rural do Rio de Janeiro, pelo apoio ao estudo.

\section{BIBLIOGRAFIA CITADA}

Baldock, J. A.; Oades, J.M.; Nelson, P.M.; Skene, T.M.; Golchin, A.; Clarke, P. 1997. Assessing the extent of decomposition of natural organic materials using solid-state 13C NMR spectroscopy. Australian Journal of Soil Research, 35: 1061-1083.

Bayer, C.; Mielniczuk, J.; Martin-Neto, L.; Ernani, P.R. 2002. Stocks and humification degree of organic matter fractions as affected by no-tillage on a subtropical soil. Plant and Soil, 238: 133-140.

Benites, V.M.; Madari, B.; Machado, P.L.O.A. 2003. Extração e fracionamento quantitativo de substâncias húmicas do solo: um procedimento simplificado de baixo custo. Comunicado Técnico 16, EMBRAPA Solos, Rio de Janeiro, Rio de Janairo. 7pp.

Benites, V.M.; Sá, E.; Schaefer, C.E.; Novotny, E.H.; Reis, E.; Ker, J.K. 2005. Properties of black soil humic acids from high altitude rock complexes in Brazil. Geoderma, 127: 104-113.
Bravard, S.; Righi, D. 1991. Characterization of fulvic and humic acids from na Oxisol-Spodosol toposequence of Amazonia, Brazil. Geoderma, 48: 151-162.

Canellas, L.P.; Santos, G.A.; Aamaral-Sobrinho; N.M.B. 1999. Reações da matéria orgânica. In: Santos, G.A.; Camargo, F.A.O. (Eds). Fundamentos da matéria orgânica do solo: ecossistemas tropicais e subtropicais. Gênesis, Porto Alegre, Rio Grande do Sul. p. 6990.

Canellas, L.P.; Velloso, A.C.X.; Marciano, C.R.; Ramalho, J.F.G.P.; Rumjanek, V.M.; Resende, C. E.; Santos, G.A. 2003. Propriedades químicas de um Cambissolo cultivado com canade-açúcar, com preservação do palhiço e adição de vinhaça por longo tempo. Revista Brasileira de Ciência do Solo, 27: 935-944.

Cunha, T.J.F. 2005. Ácidos Húmicos de Solos Escuros da Amazônia (Terra Preta do Indio). Tese de Doutorado, Universidade Federal Rural do Rio de Janeiro, Seropédica, Rio de Janairo. 139pp.

EMBRAPA.Centro Nacional de Pesquisa de Solos. 1999. Sistema brasileiro de classificação de solos. EMBRAPA - Serviço de Produção de Informação, (EMBRAPA-CNPS. Documentos, 5), Brasília, Distrito Federal. 412pp.

Francioso, O.; Ciavatta, C.; Montecchio, D.; Tugnoli, V.; SánchezCortéz, S.; Gessa, C. 2003. Quantitative estimation of peat, brown coal and lignite humic acids using chemical parameters, 1H-NMR and DTA analyses. Bioresource Technology, 88: 189195.

Glaser, B.; Balashov, E.; Haumaier, L.; Guggenberger, G.; Zech, W. 2000. Black carbon in density fractions of anthropogenic soils of the Brazilian Amazon region. Organic Geochemistry, 31(7-8): 669-678.

Golchin, A.; Clarke, P.; Baldock, J.A.; Hogashi, T.; Skejemtad, J.O.; Oades, J.M. 1997. The effects of vegetation and burning on the chemical composition of soil organic matter in a volcanic ash soil as shown by 13C NMR spectroscopy: I whole soil and humic acid fraction. Geoderma, 76: 155-174.

Kern, D.C.; Kämpf, N. 1989. Antigos assentamentos indígenas na formação de solos com terra preta arqueológica na região de Oriximiná - Pará. Revista Brasileira de Ciência do Solo, 13: 219225.

Kramer, R.W.; Kujawinski, E.B.; Hatcher, P.G. 2004. Identification of Black Carbon Derived Structures in a Volcanic Ash Soil Humic Acid by Fourier Transform Ion Cyclotron Resonance Mass Spectrometry. Enviro. Sci. Tech, 38: 3387-3395.

Lemos, R.C.; Santos, R.D. 1996. Manual de descrição e coleta de solo no campo. Sociedade Brasileira de Ciência do Solo, Campinas, São Paulo. 84pp.

Lima, H.N. 2001. Gênese, química e micromorfologia de solos da Amazônia Ocidental. Tese de Doutorado, Universidade Federal de Viçosa, Minas Gerais. 176pp.

Machado, P.L.O.A. 1999. Método para a extração de substâncias húmicas de solo: ácido húmico e ácido fúlvico. Comunicado Técnico 1, Embrapa Solos, Rio de Janeiro, Rio de Janeiro. 4pp.

Pérez, M.G.; Martin-Neto, L.; Saab, S.C.; Novotny, E.H.; Milori, D.B.P.; Bagnato, V.S.; Colnago, L.A.; Melo, W.J.; Knicker, H 2004. Characterization of humic acids from a Brazilian Oxisol 
under different tillage systems by EPR, 13C NMR, FTIR and fluorescence spectroscopy, Geoderma, 118: 181-190.

Provenzano, M.R.; Senesi, N. 1999. Thermal properties of standard and reference humic substances by differential scanning calorimetry. Journal of Anal. Colorimetry, 57: 517-526.

Rice, J.A.; MacCarthy, P. 1991. Statistical evaluation of the elemental composition of humic substances. Organic Geochemistry, 17: 635648.

Rossel, R.A.; Andriulo, A.E.; Schnitzer, M.; Crespo, M.B.; Miglierina, A.M. 1989. Humic acids properties of an Argiudoll soil under two tillage systems. The Science of the Total Environment, 81/82: 391-400.

Schmid, E.M.; Skjemstad, J.O.; Glaser, B.; Knicker, H.; KögelKnabner. 2002. Detection of charred organic matter in soils from a Neolithic settlement in Southern Bavaria, Germany. Geoderma, 107(1/2): 71-91.

Schnitzer, M.; Gupta, U.C. 1965. Determination of acidity in soil organic matter. Soil Science Society of America Proceedings, 27: 274-277.

Schnitzer, M.; Khan, S.U. 1972. Humic substances in the environment. Marcel Decker, New York, USA. 220pp.

Smith, N.J.H. 1980. Anthrosols and human carring capacity in Amazonia. Annals of The Association of American Geographers, 70: 553-566.

Sombroek, W. G. 1966. Amazon soils. A reconnaissance of the soils of the Brazilian Amazon region. Wageningen: Centre for Agricultural Publication and Documentation. 292pp.
Stevenson, F.J. 1994. Humus chemistry: genesis, composition, reactions. 2. ed. John Willey, New York, USA. 496pp.

Swift, R.S. 1996. Organic matter characterization. In: Sparks, D.L.; Page, A.L.; Helmke, P.A.; Loeppert, R.H.; Soltanpour, P.N.; Tabatabai, M.A.; Johnston, C.T.; Sumner, M.E. (Eds.) Methods of soil analysis: chimical methods. Vol. 3. Soil Science Society of America; American Society of Agronomy, Madison. (SSSA. Book Series, 5). p. 1011-1020.

Woods, W.I.; McCann, J.M. 2001. El origen y persistencia de las tierras negras de la Amazonía. In: Hiraoka, M.; Mora, S. (Eds). Desarrollo Sostentible en la Amazonía, Abya Ayala, Quito, Ecuador. p. 23-30.

Yoemans, J.C.; Bremner, J.M. 1988. A rapid and precise method for routine determination of organic carbon in soil. Commun. Soil Sci. Plant Anal, 19(13): 1467-1476.

Zech, W.; Haumaier, L.; Hempfling, R. 1990. Ecological aspects of soil organic matter in tropical land use. In: McCarthy, P.; Clapp, C.E.; Malcolm, R.L.; Blomm, P.R. (Eds). Humic substances in soil and crop sciences: selected Reedings.: ASA: SSSA, Madison, USA. p.187-201.

Recebido em 01/07/2005

Aceito em 01/12/2006 